\title{
Childhood BMI trajectories and the risk of developing young adult-onset diabetes
}

\author{
N. Lammi • E. Moltchanova • P. A. Blomstedt • \\ J. Tuomilehto • J. G. Eriksson • M. Karvonen
}

Received: 7 May 2008 / Accepted: 3 December 2008 /Published online: 8 January 2009

(C) Springer-Verlag 2008

\begin{abstract}
Aims/hypothesis The aim of this study was to examine the effects of childhood BMI growth dynamics on the risk of developing young adult-onset type 1 and type 2 diabetes. Methods Finnish national healthcare registers were used to identify individuals with diabetes diagnosed between 1992 and 1996 at 15-39 years of age. Non-diabetic control participants were chosen from the National Population Registry. Anthropometric measurements were obtained from the original child welfare clinic records. Only the case-control pairs with sufficient growth data recorded were included in the analyses $(218 / 1,388$ for type 1 diabetes [16\%] and 64/1,121 for type 2 diabetes [6\%]). Two developmental stages in BMI growth (the points of infancy maximum BMI and the BMI rebound) were examined, and conditional logistic regression was applied to the variables of interest.
\end{abstract}

N. Lammi $(\bowtie) \cdot$ E. Moltchanova $\cdot$ P. A. Blomstedt

J. Tuomilehto · J. G. Eriksson $\cdot$ M. Karvonen

Diabetes Unit, Department of Health Promotion and Chronic

Disease Prevention, National Public Health Institute,

Mannerheimintie 166,

00300 Helsinki, Finland

e-mail: niina.lammi@helsinki.fi

e-mail: niina.lammi@ktl.fi

J. Tuomilehto

Department of Public Health, University of Helsinki,

Helsinki, Finland

J. G. Eriksson

Department of General Practice and Primary Health Care,

University of Helsinki,

Helsinki, Finland

J. G. Eriksson

Vasa Central Hospital,

Vasa, Finland
Results The risk for type 1 diabetes increased 1.19-fold per $1 \mathrm{~kg} / \mathrm{m}^{2}$ rise in the infancy maximum BMI $(p=0.02)$. In addition, there was a 1.77 -fold increase in the risk for type 2 diabetes per $1 \mathrm{~kg} / \mathrm{m}^{2}$ rise in the level of BMI at the BMI rebound $(p=0.04)$. Higher values of BMI at these points corresponded to a larger BMI gain from birth to that developmental stage. Age at the infancy maximum BMI or age at the BMI rebound did not affect the risk for either type of diabetes.

Conclusions/interpretation The BMI gain in infancy among individuals who subsequently developed young adult-onset type 1 diabetes was faster than that of those who remained healthy. The excess BMI gain in individuals who developed young adult-onset type 2 diabetes could already be seen during early childhood.

Keywords Body mass index · Child development .

Diabetes mellitus $\cdot$ Epidemiology $\cdot$ Growth

\section{Abbreviation \\ ICP Infancy-childhood-puberty}

\section{Introduction}

Type 1 and type 2 diabetes can be diagnosed at any age, a characteristic that the old terms 'juvenile-onset' and 'adultonset' diabetes did not convey. A significant proportion of new cases of type 1 diabetes are diagnosed after puberty [1, 2], and early-onset type 2 diabetes has become an important public health problem worldwide [3]. In Finland, the incidence of both type 1 and type 2 diabetes is increasing rapidly among 15-39-year-old individuals [4]. The possible 
environmental exposures behind this increase need to be identified.

It has been shown that individuals with type 2 diabetes not only have lower birthweight than their peers, but also have a lower BMI until the age of 2 years $[5,6]$. This situation is reversed later in childhood, as the BMI rebound (the period after infancy when BMI starts to increase) occurs earlier in persons who later develop type 2 diabetes $[5,7]$; these individuals have an accelerated increase in BMI thereafter $[5,8]$.

The role of childhood growth has also been studied in relation to the risk of developing type 1 diabetes. People with childhood-onset type 1 diabetes are heavier [9-11] and taller [10-12] during infancy (which here is defined as the first 3 years of a child's life), and seem to gain weight faster during their first years [13]. The impacts of childhood growth and weight gain on adult-onset type 1 diabetes are still unknown, because in previous studies investigating the effects of childhood growth on type 1 diabetes all participants had been diagnosed under the age of 16 years [9-13].

In the present case-control study we examined the effects of childhood BMI growth dynamics on the risks of developing type 1 and type 2 diabetes diagnosed at 1539 years old. We chose BMI as the explanatory variable, as it takes both height and weight into account. The infancychildhood-puberty (ICP) model for human growth [14], which divides growth into three components (infancy, 03 years; childhood, 3-11 years; puberty, >11 years), was used as a starting point. Once the neonatal period is over, the BMI of a child normally increases. After reaching a certain point, referred to here as the infancy maximum BMI, the BMI starts to decrease. This happens at about the time the child learns to walk. At between 3 and 11 years old the BMI begins to increase again, referred to here as the BMI rebound. Our analysis was focused on these two turning points (the infancy maximum BMI and the BMI rebound; Fig. 1) in the BMI growth pattern. This approach

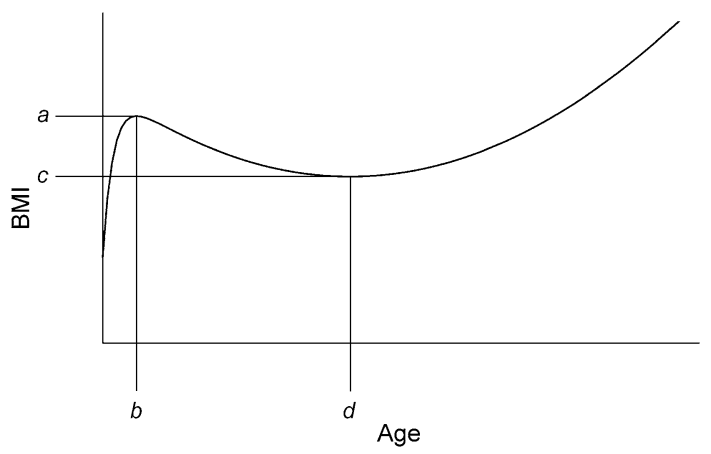

Fig. 1 BMI growth curve illustrating the BMI maximum and the BMI minimum. $a$ the maximum BMI value in the infancy component; $b$ the age at which the maximum BMI value in the infancy component is reached; $c$ the minimum BMI value in the childhood component (the BMI rebound); $d$ the age at which the minimum BMI value in the childhood component is reached allowed us to compare the BMIs of case and control individuals at the same stage of development, despite individual variation in the timing of these turning points. The chosen turning points can therefore be seen as an alternative to comparing BMI levels at predefined ages. The average height and weight of children and adolescents varies depending on the age cohort, and the average agespecific BMI has recently been increasing [15]. Therefore, we compared the BMI gain of individuals with diabetes with that of birth date- and birth place-matched controls, instead of utilising the Finnish growth standards. Because it was possible to adjust the results for birthweight, higher or lower BMI at a specific turning point can be interpreted as larger or smaller BMI gain, respectively, from birth to that developmental stage.

\section{Methods}

Study participants All participants were resident in Finland and were diagnosed with type 1 or type 2 diabetes at 15 39 years old between the years 1992 and 1996. Finnish national healthcare registers were used to identify diabetic patients. Information on purchased medication was obtained from the Drug Prescription Register; a list of persons entitled to free-of-charge medication for diabetes was acquired from the Drug Reimbursement Register; and hospital discharge diagnoses referring to diabetes were obtained from the Hospital Discharge Register. In addition, the diabetes nurses in hospitals and primary healthcare clinics nationwide reported new cases of diabetes (at 15-39 years old) to the National Public Health Institute using standardised forms. Information obtained from these four data sources was linked using the personal identification number assigned to every Finnish resident. The inclusion criterion was a uniform diagnosis from a minimum of two data sources. Classification of diabetes was based on physician-assigned diagnosis (the Hospital Discharge Register) and medication (the Drug Prescription Register). The diagnoses made in hospitals and recorded in the Hospital Discharge Register were based on clinical characteristics, and in unclear cases also on C-peptide and autoantibody measurements. Medical records, including information on clinical characteristics and medication, were reviewed for individuals whose diagnosis remained unclear after examining the data in the register. The American Diabetes Association criteria [16] were used for the classification of diabetes. The data sources and the method of classification have been described in detail previously [17]. In total, 1,388 cases of type 1 diabetes and 1,121 cases of type 2 diabetes were identified and chosen as the diabetes cases for this study. The age-standardised incidence of diabetes in the years $1992-1996$ was 15.9 per $100,000 /$ year for type 1 diabetes and 11.8 per $100,000 /$ year for type 2 
diabetes in the Finns aged 15-39 years who were examined [17]. The study population was almost exclusively of Europid origin. Individuals with gestational diabetes $(n=1,268)$, secondary forms of diabetes $(n=216)$ and those whose diabetes type could not be defined $(n=485)$ were excluded. Two control individuals matched by age, sex and place of birth were chosen for every case with type 1 or type 2 diabetes from the National Population Register. The nondiabetic status of the control individuals was confirmed by computer linkage to the Hospital Discharge Register and the Drug Prescription Register.

Child welfare and school healthcare records The health of Finnish children is followed up within the primary healthcare system in child welfare clinics, where children attend regular examinations by public health nurses and doctors until the age of 7 years. Thereafter, these examinations are continued within the school healthcare system until the end of the compulsory education at 15 years old. An essential part of this follow-up is the measurements of height and weight of the children; these are documented in the child welfare clinic records. These records also include information on the duration of breastfeeding and on the body size and health of the child as a newborn infant. The child welfare clinic records were collected across Finland nationwide for patients with type 1 and type 2 diabetes and for the control participants. The records were acquired from the archives of health centres and the archives of municipalities from the 200 largest municipalities in Finland (population $>3,500$ ). The records were traced based on the place of birth obtained from the National Population Registry.

Child welfare clinic records were obtained for 4,440 persons, for whom it was possible to form 607 case-control pairs with type 1 diabetes and 324 case-control pairs with type 2 diabetes, with child welfare clinic records available for the diabetes patient and for at least one of the controls. Twenty individuals were excluded from the study as they had no valid anthropometric observations, and 18 individuals were excluded because they were simultaneously cases and controls. Only the case-control pairs with sufficient recorded growth data were included in the analyses (218 case-control pairs of type 1 diabetes (16\%) from the original 1,388 pairs, and 64 case-control pairs of type 2 diabetes $(6 \%)$ from the original 1,121 pairs; Fig. 2). In total, the number of anthropometric measurements was 53,252 . The study plan was approved by the National Advisory Board on Health Care Ethics.

Statistical methods Conditional logistic regression [18] was used to evaluate the effect of the properties of individual BMI growth curves on the risk of developing type 1 or type 2 diabetes in young adulthood. To define these properties, the ICP model for human growth [14] was used as a

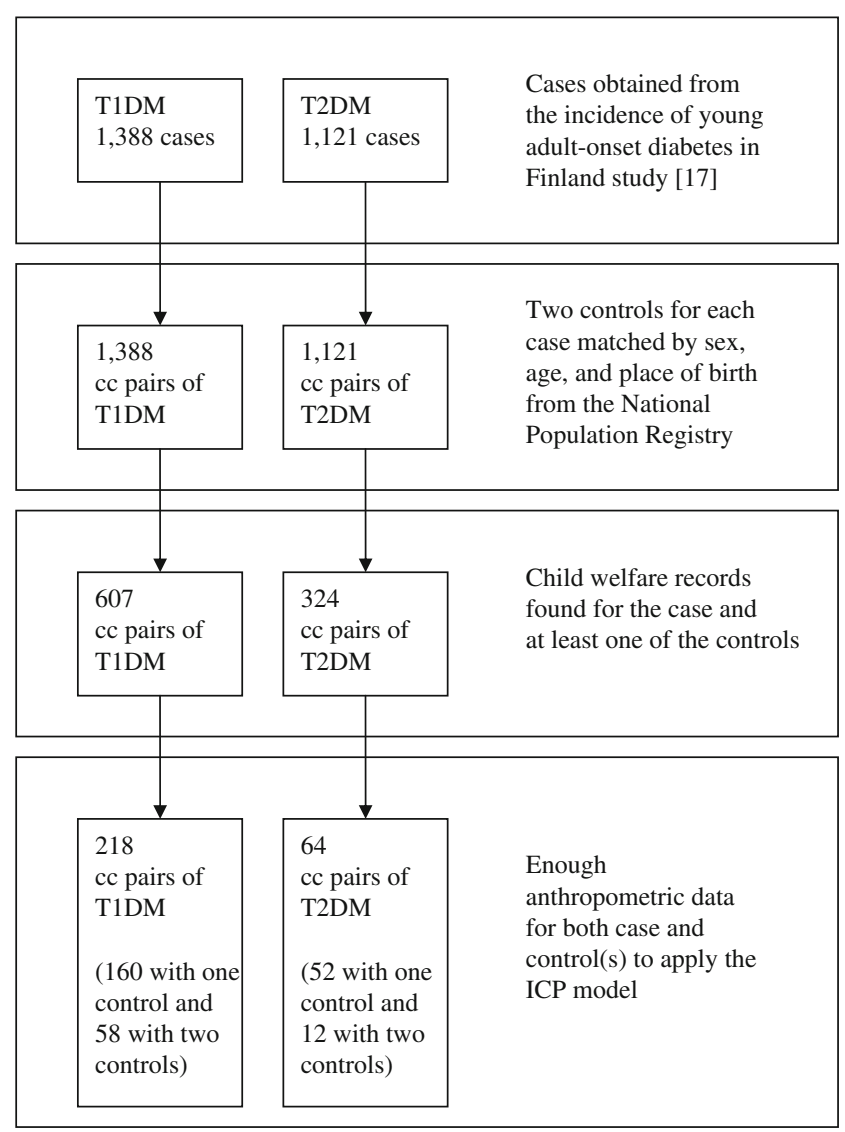

Fig. 2 Method of data collection. cc, case-control; T1DM, type 1 diabetes; T2DM, type 2 diabetes

starting point. This model breaks down growth into three additive components (infancy, 0-3 years; childhood, 311 years; and puberty $>11$ years), where each component is a separate parametric function of age. From each individual growth curve a set of characteristics was extracted to be used as explanatory variables in conditional logistic regression. Only the first two components were considered in the analyses due to the scarcity of measurements after 11 years of age. The extracted characteristics were as follows (see Fig. 1): (a) the maximum BMI value in the infancy component (the BMI level at which the BMI starts to decrease between 0 and 3 years old); (b) the age at which the maximum BMI value in the infancy component is reached; (c) the minimum BMI value in the childhood component (the BMI rebound); (d) the age at which the minimum BMI value in the childhood component is reached.

The results were adjusted for birthweight. Estimation of the infancy component was not possible for most individuals because there was too much variation in the BMI values under the age of 3 years. Therefore, observed rather than estimated values were extracted from this component. 
Table 1 Sex ratio and characteristics of the case-control pairs

\begin{tabular}{|c|c|c|c|c|}
\hline \multirow[t]{2}{*}{ Characteristic } & \multicolumn{2}{|c|}{ Type 1 diabetes } & \multicolumn{2}{|c|}{ Type 2 diabetes } \\
\hline & Cases & Controls & Cases & Controls \\
\hline$n$ & 218 & 276 & 64 & 76 \\
\hline Sex (male/female) & $146 / 72$ & $184 / 92$ & $39 / 25$ & $45 / 31$ \\
\hline Age at diagnosis (years) & $26.5(7.1)$ & - & $34.5(4.4)$ & - \\
\hline Birthweight (kg) & $3.55(0.55)$ & $3.56(0.50)$ & $3.39(0.62)$ & $3.50(0.54)$ \\
\hline Maximum BMI at $0-3$ years old $\left(\mathrm{kg} / \mathrm{m}^{2}\right)$ & $18.55(2.61)$ & $18.25(1.83)$ & $18.34(1.98)$ & $18.26(2.07)$ \\
\hline Age at maximum BMI (years) & $0.96(0.57)$ & $0.89(0.53)$ & $0.97(0.62)$ & $0.88(0.50)$ \\
\hline Minimum BMI at $3-11$ years old $\left(\mathrm{kg} / \mathrm{m}^{2}\right)$ & $15.46(1.42)$ & $15.31(1.24)$ & $15.88(1.92)$ & $15.27(1.24)$ \\
\hline Age at minimum BMI (years) & $6.2(1.4)$ & $6.3(1.4)$ & $6.3(1.6)$ & $6.7(1.4)$ \\
\hline
\end{tabular}

Values are means (SD)

All analyses were done in the $\mathrm{R}$ environment for statistical computing [19]. The Survival package [20] was used for conditional logistic regression.

\section{Results}

The sample numbers and characteristics of the case-control pairs examined are presented in Table 1. Sixty-seven per cent of the case-control pairs of type 1 diabetes and $61 \%$ of the case-control pairs of type 2 diabetes were men. Although the individuals with type 1 diabetes and type 2 diabetes were all diagnosed at between 15 and 39 years old, those with type 1 diabetes had a lower mean age of diagnosis than those with type 2 diabetes ( 26.5 vs 34.5 years old, respectively). The childhood BMI patterns of the diabetic individuals compared with their controls are presented in Fig. 3a (type 1 diabetes) and Fig. 3b (type 2 diabetes). Visually, the BMI growth trajectories of the patients with young adult-onset type 1 diabetes were close to those of their controls, while the growth patterns of the patients with young adult-onset type 2 diabetes were prominently aberrant. The average number of measurements (height and weight) per child was 7.3 (range $0-32$ ) between 0 and 3 years old, 5.7 (range $0-22$ ) between 3 and 11 years old, and 3.8 (range $0-19$ ) for $>11$ years old.

Type 1 diabetes The ORs describing the effect sizes for the childhood BMI characteristics examined for the risk of developing young adult-onset type 1 diabetes are presented in Table 2. The analysis, which included 218 case-control pairs (160 with one control and 58 with two controls) indicated that the odds for type 1 diabetes increased 1.19-fold for every $1 \mathrm{~kg} / \mathrm{m}^{2}$ increase in the infancy maximum BMI under the age of 3 years $(p=0.02)$. The CI was large $(95 \%$ CI 1.03-1.36). There were no age differences in the timing of the infancy maximum BMI. In addition, the BMI at the BMI rebound at between 3 and 11 years old was similar in individuals with type 1 diabetes and in control participants. The age for the BMI rebound at between 3 and 11 years old was slightly, but not significantly, lower in individuals with
Fig. 3 BMI growth curves of cases of type 1 diabetes (a) or type 2 diabetes (b) and their controls. Case, dashed line; controls, solid line; individual observations, grey circles
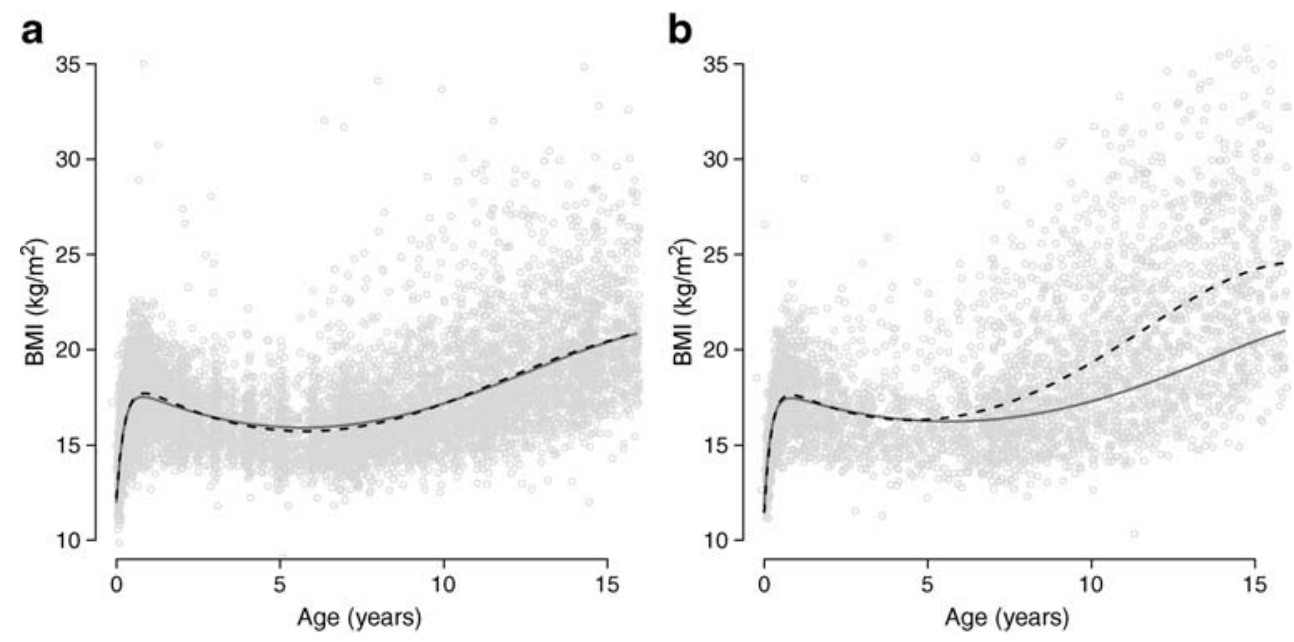
type 1 diabetes. Adjusting the BMI analysis for birthweight did not significantly change the results (Table 2).

Type 2 diabetes The ORs for the risk of developing young adult-onset type 2 diabetes according to childhood BMI characteristics are presented in Table 2. The analysis of young adult-onset type 2 diabetes was based on data from 64 case-control pairs (52 with one control and 12 with two controls). The level of infancy maximum BMI and age at the infancy maximum BMI under the age of 3 years were similar for the cases and the controls. The risk of developing type 2 diabetes was strongly affected by BMI at the BMI rebound at between 3 and 11 years old. There was a 1.77 -fold increase in the odds for the risk of developing type 2 diabetes with a gain of $1 \mathrm{~kg} / \mathrm{m}^{2}$ in the minimum BMI at between 3 and 11 years old $(p=0.04)$. The CI was large (95\% CI 1.04-3.00). The effect became slightly stronger when adjusted for birthweight (OR 1.87, 95\% CI 1.04-3.37; $p=0.04$ ). Among the individuals with type 2 diabetes, the BMI rebound occurred at a younger age, but the effect was not statistically significant. The excess BMI gain in individuals who developed young adult-onset type 2 diabetes had already begun in early childhood, after the age at which the BMI rebound occurred (Fig. 3b).

\section{Discussion}

In this case-control study, BMI trajectories from birth to 11 years of age were examined among individuals with young adult-onset type 1 and type 2 diabetes. The results showed that higher infancy maximum BMI before the age of 3 years increased the risk for young adult-onset type 1 diabetes independent of birthweight. The large BMI gain during infancy among individuals who later developed type 1 diabetes may be due to common genetic traits between growth and type 1 diabetes, or to nutritional factors. Another major finding was the markedly elevated risk for young adult-onset type 2 diabetes in individuals whose BMI was high at the time of BMI rebound. This indicates that although individuals with type 2 diabetes do not have high BMI at birth or during infancy, the excess BMI gain among them began early in childhood and was already apparent after 3 years of age.

Although the cases of type 1 and type 2 diabetes were collected nationwide in this study, only those case-control pairs for whom the child welfare record had sufficient data available on growth could be included in the analysis of BMI growth dynamics. As a result, only $16 \%$ of the original case-control pairs of type 1 diabetes and $6 \%$ of the original case-control pairs of type 2 diabetes could be included in the present analysis. The reason for the lower number of case-control pairs included for type 2 diabetes is their older average age compared with the case-control pairs for type 1 diabetes; child welfare clinic records were more difficult to trace for older age groups. Because of the retrospective approach of this study, it is possible that individuals with aberrant growth patterns were measured more frequently than individuals who were growing on the standard curve, and the former were therefore included in the study more often. The case-control setting of this study is efficient in reducing the possible error caused by the uneven frequency of measurements, because the reason for the exclusion of a case-control pair was equally often caused by shortage of data for the case participants. This indicates that there was a corresponding proportion of case and control individuals who were measured especially frequently in the study dataset (i.e. possibly aberrantly growing). However, due to the fact that the number of case-control pairs with

Table 2 The ORs for type 1 diabetes and type 2 diabetes diagnosed at between 15 and 39 years old according to childhood BMI characteristics

\begin{tabular}{|c|c|c|c|c|c|}
\hline \multirow[t]{2}{*}{ Type of diabetes } & \multirow[t]{2}{*}{ Examined risk factor } & \multicolumn{2}{|c|}{ Not adjusted for birthweight } & \multicolumn{2}{|c|}{ Adjusted for birthweight } \\
\hline & & OR $(95 \% \mathrm{CI})$ & $p$ value & OR $(95 \% \mathrm{CI})$ & $p$ value \\
\hline \multicolumn{6}{|l|}{ Type $1^{\mathrm{a}}$} \\
\hline $\mathrm{a}$ & Maximum BMI under the age of 3 years per $1 \mathrm{~kg} / \mathrm{m}^{2}$ & $1.19(1.03-1.36)$ & 0.02 & $1.21(1.05-1.41)$ & 0.01 \\
\hline $\mathrm{b}$ & Age at maximum BMI per year & $1.16(0.82-1.65)$ & NS & $1.14(0.80-1.64)$ & NS \\
\hline $\mathrm{c}$ & Minimum BMI at $3-11$ years old per $1 \mathrm{~kg} / \mathrm{m}^{2}$ & $0.90(0.73-1.10)$ & NS & $0.88(0.70-1.09)$ & NS \\
\hline $\mathrm{d}$ & Age at minimum BMI per year & $0.87(0.75-1.02)$ & NS & $0.88(0.75-1.03)$ & NS \\
\hline \multicolumn{6}{|c|}{ 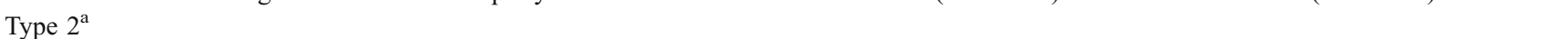 } \\
\hline $\mathrm{a}$ & Maximum BMI under the age of 3 years per $1 \mathrm{~kg} / \mathrm{m}^{2}$ & $0.91(0.59-1.41)$ & NS & $0.93(0.59-1.48)$ & NS \\
\hline $\mathrm{b}$ & Age at maximum BMI per year & $0.69(0.28-1.70)$ & NS & $0.79(0.29-2.07)$ & NS \\
\hline $\mathrm{c}$ & Minimum BMI at $3-11$ years old per $1 \mathrm{~kg} / \mathrm{m}^{2}$ & $1.77(1.04-3.00)$ & 0.04 & $1.87(1.04-3.37)$ & 0.04 \\
\hline $\mathrm{d}$ & Age at minimum BMI per year & $0.75(0.53-1.06)$ & NS & $0.75(0.52-1.09)$ & NS \\
\hline
\end{tabular}

Results for 218 case-control pairs of type 1 diabetes and 64 case-control pairs of type 2 diabetes

${ }^{a}$ Letters refer to illustration in Fig. 1 
adequate data available to estimate the growth curve was reduced to 218 pairs of type 1 diabetes and 64 pairs of type 2 diabetes, the statistical power was reduced, i.e. the nonsignificant effects reported here do not rule out the possibility that significant results could be obtained with a larger dataset.

The observed increase in the risk of young adult-onset type 1 diabetes among individuals with high infancy BMI is consistent with previous findings among individuals with childhood-onset type 1 diabetes $[11,12]$. The effect of high BMI among individuals with young adult-onset type 1 diabetes was, however, weaker than among individuals with childhood-onset type 1 diabetes [11, 12]. Unlike in childhood-onset type 1 diabetes [11], the differences in BMI between individuals with young adult-onset type 1 diabetes and control individuals levelled out by the time of the BMI rebound. The timing of the two turning points did not affect the risk of young adult-onset type 1 diabetes. Therefore the larger BMI gain from birth to the infancy maximum BMI can also be interpreted as faster BMI gain in infancy among the individuals who were later diagnosed with type 1 diabetes.

In accordance with earlier observations [5, 21], the average age of the BMI rebound in this study was slightly, but not significantly, lower in individuals with type 2 diabetes. In addition, our results showed that high BMI at the BMI rebound at age 3-11 years increased the risk for type 2 diabetes, although high BMI earlier at the infancy maximum BMI did not have an effect. This is in accordance with earlier findings that the increase in BMI among individuals with type 2 diabetes is slow until 2 years of age [6], but these individuals have a higher BMI at 715 years old [8]. In this study, among the individuals with young adult-onset type 2 diabetes the rapid increase in BMI began at a very young age after the age of the BMI rebound. Rapid increase of BMI at adolescence is strongly associated with central obesity in adults [22], which in turn markedly increases the risk of type 2 diabetes [23]. The fast childhood BMI growth rate in individuals with type 2 diabetes also suggests that excessive energy intake in childhood and adolescence tends to persist into adult age. Primary prevention of type 2 diabetes should therefore be directed against childhood and adolescent obesity.

Conclusions The BMI gain in infancy among individuals with young adult-onset type 1 diabetes is faster than among those remaining healthy. The excess BMI gain in individuals with young adult-onset type 2 diabetes can be demonstrated in early childhood.

Acknowledgements We thank O. Taskinen, N. Schreier, S. Rosten and our data recording team for the efforts with data collection and data management. This work was funded by the National Institutes of Health (grant no. DK062374-01A1) and the Academy of Finland (grants no. 207008 and no. 214111).

Duality of interest The authors declare that there is no duality of interest associated with this manuscript.

\section{References}

1. Melton LJ, Palumbo PJ, Chu CP (1983) Incidence of diabetes mellitus by clinical type. Diabetes Care 6:75-86

2. Laakso M, Pyorälä K (1985) Age of onset and type of diabetes. Diabetes Care 8:114-117

3. International Diabetes Federation (2007) Diabetes atlas, 3rd edn. International Diabetes Federation, Brussels

4. Lammi N, Blomstedt PA, Moltchanova E, Eriksson JG, Tuomilehto J, Karvonen M (2008) Marked temporal increase in the incidence of type 1 and type 2 diabetes among young adults in Finland. Diabetologia 51:897-899

5. Bhargava SK, Sachdev HS, Fall CH et al (2004) Relation of serial changes in childhood body-mass index to impaired glucose tolerance in young adulthood. N Engl J Med 350:865-875

6. Eriksson J, Osmond C, Kajantie E, Forsen T, Barker D (2006) Patterns of growth among children who later develop type 2 diabetes or its risk factors. Diabetologia 49:2853-2858

7. Wadsworth M, Butterworth S, Marmot M, Ecob R, Hardy R (2005) Early growth and type 2 diabetes: evidence from the 1946 British birth cohort. Diabetologia 48:2505-2510

8. Forsen T, Eriksson J, Tuomilehto J, Reunanen A, Osmond C, Barker D (2000) The fetal and childhood growth of persons who develop type 2 diabetes. Ann Intern Med 133:176-182

9. Hyppönen E, Kenward MG, Virtanen SM et al (1999) Infant feeding, early weight gain, and risk of type 1 diabetes. Childhood Diabetes in Finland (DiMe) Study Group. Diabetes Care 22:19611965

10. EURODIAB Substudy 2 Study Group (2002) Rapid early growth is associated with increased risk of childhood type 1 diabetes in various European populations. Diabetes Care 25:1755-1760

11. Hyppönen E, Virtanen SM, Kenward MG, Knip M, Åkerblom HK (2000) Obesity, increased linear growth, and risk of type 1 diabetes in children. Diabetes Care 23:1755-1760

12. Svensson J, Carstensen B, Mortensen H, Borch-Johnsen K, The Danish Study Group of Childhood Diabetes (2007) Growth in the first year of life and the risk of type 1 diabetes in a Danish population. Pediatr Perinatal Epidemiol 21:44-48

13. Johansson C, Samuelsson U, Ludvigsson J (1994) A high weight gain early in life is associated with an increased risk of type 1 (insulin-dependent) diabetes mellitus. Diabetologia 37:91-94

14. Karlberg J (1989) A biologically-oriented mathematical model (ICP) for human growth. Acta Paediatr Scand Suppl 350:70-94

15. Kautiainen S, Rimpelä A, Vikat A, Virtanen SM (2002) Secular trends in overweight and obesity among Finnish adolescents in 1977-1999. Int J Obes Relat Metab Disord 26:544-552

16. American Diabetes Association (2006) Diagnosis and classification of diabetes mellitus. Diabetes Care 29(Suppl 1):S43-S48

17. Lammi N, Taskinen O, Moltchanova E et al (2007) A high incidence of type 1 diabetes and an alarming increase in the incidence of type 2 diabetes among young adults in Finland during 1992 to 1996. Diabetologia 50:1393-1400

18. Hosmer D, Lemeshow S (2000) Applied logistic regression, 2nd edn. Wiley, New York 
19. R Development Core Team (2006) R: A language and environment for statistical computing. Available from www.R-project.org, accessed 4 November 2008

20. Therneau T, Lumley $T$ (2008) Survival: survival analysis, including penalised likelihood. R package version 2.34. Available from www.r-project.org, accessed 4 November 2008

21. Eriksson JG, Forsen T, Tuomilehto J, Osmond C, Barker DJ (2003) Early adiposity rebound in childhood and risk of type 2 diabetes in adult life. Diabetologia 46:190-194
22. Sachdev HS, Fall CH, Osmond $\mathrm{C}$ et al (2005) Anthropometric indicators of body composition in young adults: relation to size at birth and serial measurements of body mass index in childhood in the New Delhi birth cohort. Am J Clin Nutr 82: 456-466

23. Janssen I, Katzmarzyk PT, Ross R (2002) Body mass index, waist circumference, and health risk: evidence in support of current National Institutes of Health guidelines. Arch Intern Med 162: 2074-2079 